\title{
Complementariedad terapéutica en el trastorno depresivo mayor con síntomas psicóticos
}

\section{Therapeutic complementarity in major depressive disorder with psychotic symptoms}

\author{
Xavier Fabricio Reyes Trelles ${ }^{1 *}$, Cristhian Javier Rivera Tapia ${ }^{2}$, Braulio Andrés Elizalde Martínez ${ }^{3}$, Álvaro \\ Fabián Auquilla Guzmán ${ }^{2}$, Yolanda María Malavé Pérez ${ }^{2}$ \\ ${ }^{1}$ Ministerio de Inclusión Económica y Social, Ecuador \\ ${ }^{2}$ Universidad Católica de Cuenca, Ecuador \\ ${ }^{3}$ Fundación de Desarrollo Social de El Oro - FUDESO, Ecuador \\ *fabricioreyestrelles@gmail.com
}

DOI: https://doi.org/10.26871/killkana_salud.v4i3.627

\begin{abstract}
Resumen
Contexto: La población clínica con depresión mayor y síntomas psicóticos representa un desafío para la atención tanto psicológica como psiquiátrica, su complejidad inherente resalta la necesidad de diseño de protocolos de tratamiento complementarios, la efectividad en estos tipos de tratamiento implica no solo la coordinación de objetivos y tiempos de intervención, sino también el manejo de un lenguaje común de tratamiento y la cooperación interdisciplinar. Objetivo: la investigación representa una revisión descriptiva alrededor de los procesos de abordaje psicoterapéutico y farmacológico, para lograr una comprensión abarcativa de los recursos terapéuticos efectivos actuales, además de, establecer una propuesta de tratamiento con base en los datos recabados. Procedimiento: se realizó una búsqueda y revisión de la literatura científica actualizada a la última década, por medio de las bases de datos Scopus y ResearchGate, seleccionando investigaciones empíricas, estudios de caso clínico y revisiones sistemáticas. Resultados: en tanto al abordaje psicoterapéutico el modelo cognitivo-conductual figura como el principal recurso de intervenciones fundamentadas en evidencia, también, se consideran necesarias intervenciones de corte psicosocial y de rehabilitación de funciones cognitivas. A partir de los mismos se estructuró una planificación psicoterapéutica específica al trastorno. La propuesta desde la farmacología conlleva el uso de antidepresivos inhibidores selectivos de la recaptación de serotonina y antipsicóticos de segunda generación. Conclusiones: La complementariedad terapéutica ideal implica una conjunción coordinada de las intervenciones, así como un abordaje personalizado que se ajusta a los ritmos de evolución de cada caso en particular.
\end{abstract}

Palabras clave: depresión mayor, sintomatología psicótica, psicoterapia, farmacoterapia, planificación terapéutica.

\begin{abstract}
The clinical population diagnosed with major depression and psychotic symptoms represents a challenge for both psychological and psychiatric care; its inherent complexity highlights the need to design treatment protocols using a complementary approach. The effectiveness of these types of treatment implies not only the coordination of objectives and intervention times, but also the management of a common language of treatment as well as interdisciplinary cooperation. Objective: for the research a descriptive review was carried around psychotherapeutic and pharmacological approaches within this disorder, aiming to achieve a comprehensive understanding of the current effective therapeutic resources, as well as to establish a treatment proposal based on the data collected. Procedure: a search and review of the scientific literature updated to the last decade was carried out through the Scopus and ResearchGate databases, selecting empirical research, clinical case studies and other systematic reviews of the literature. Results: the cognitive-behavioral model is the main resource for evidence-based interventions. However, psychosocial interventions and rehabilitation of cognitive functions are also considered necessary. From the results, a psychotherapeutic planning chart specific to the disorder was structured. The pharmacotherapy proposal involves the use of selective serotonin reuptake inhibitor antidepressants and second-generation antipsychotics. Conclusions: The ideal therapeutic complementarity implies a coordinated conjunction of the interventions, as well as a personalized approach that adjusts to the rhythm of evolution in each particular case.
\end{abstract}

Keywords: major depression, psychotic symptoms, psychotherapy, pharmacotherapy, therapeutic planning. 


\section{Introducción}

Los trastornos depresivos afectan a un $5 \%$ de la población general y a un $2,6 \%$ de la población infanto-juvenil en el mundo. ${ }^{1}$ según la Organización Mundial de la Salud (OMS) es la undécima causa de discapacidad mundial. ${ }^{2} \mathrm{El}$ trastorno depresivo mayor (TDM) es una patología ampliamente extendida con una prevalencia a lo largo de la vida del $15 \%$ de quienes la padecen. Se asocia con costos significativos en la calidad de vida, pérdida de productividad laboral y riesgo de mortalidad. Alrededor de dos tercios de los pacientes no logran una remisión completa, la cual es crucial para restablecer el funcionamiento y prevenir recaídas. ${ }^{3}$

De acuerdo al DSM-5, ${ }^{4}$ el TDM está caracterizado por un conjunto de síntomas de los que destacan: estado de ánimo deprimido y/o perdida de placer durante un periodo de por lo menos 15 días, causantes de un deterioro significativo. Además, toma en cuenta especificadores que hacen referencia a características asociadas al trastorno, como los síntomas psicóticos cuando existe la presencia de delirios y/o alucinaciones que, dependiendo de su contenido, pueden o no ser congruentes con el estado de ánimo.4 Como propone el Ministerio de Salud Pública del Ecuador ${ }^{5}$ se denomina como TDM con características psicóticas, cuando en un caso se cumplen los criterios del trastorno depresivo grave más cualquiera de los siguientes síntomas: 1) ideas delirantes o alucinaciones diferentes a las descritas como típicas de la esquizofrenia y 2) estupor depresivo. ${ }^{5}$

La población clínica con depresión y psicosis comórbida, representa un desafío para la atención clínica pues se asocia a múltiples problemas, incluyendo el suicidio y el deterioro funcional severo. Por consiguiente, es importante evaluar cuidadosamente las necesidades de cada paciente y adaptar la intervención a los problemas clínicos específicos que se presenten. También es importante que se intervenga en un ritmo adecuado, de modo que no se abrume al paciente y se actué cuando sea más beneficioso. Los tratamientos con medicamentos que los pacientes reciben para la depresión y la psicosis suelen reducir los síntomas, pero también pueden producir efectos adversos que repercuten negativamente en el funcionamiento. ${ }^{6}$

Para el diseño de un plan de tratamiento efectivo se debe partir de criterios fundamentales, sobre los cuales el tratamiento debe ser: basado en evidencia, reforzado por valoraciones psicométricas, complementario y personalizado. ${ }^{3}$ Es bajo estos criterios que se propone desarrollar el presente documento. Finalmente, se debe hacer hincapié en que no existe evidencia para un modelo psicoterapéutico específico en este subtipo de depresión. ${ }^{2}$

Aunando todo lo anterior el objetivo de la revisión es lograr una comprensión abarcativa de los recursos terapéuticos efectivos actuales. Posterior a la revisión descriptiva de las diferentes líneas de intervención desde los enfoques preestablecidos, esclareciendo sus pautas principales y evidenciado su efectividad, se pretende esbozar un plan de intervención psicoterapéutico actualizado y contextualizado que contemple integralmente el trastorno depresivo mayor con síntomas psicóticos. La elaboración del mismo toma especial énfasis al evidenciar limitaciones en la literatura cuando la depresión se acompaña de síntomas psicóticos, más aun, al tratar de encontrar referencias contextualizadas a nuestro entorno, teniendo en cuenta que las guías locales están enfocadas en aspectos generales.

\section{Metodología}

Se efectuó una revisión de los artículos encontrados en las bases de datos Scopus y ResearchGate, sobre la temática de intervenciones psicoterapéuticas y farmacológicas en el tratamiento de la depresión con síntomas psicóticos. Para la búsqueda en Scopus se utilizó las siguientes palabras claves: "major depresion", "psychotic feats" y "complementary treatment"; mientras que en ResearchGate se utilizaron las palabras "depresión mayor", psicosis" y "tratamiento".

Los criterios de inclusión fueron los siguientes:

- Investigaciones empíricas

- Revisiones de la literatura

- Estudios de caso clínicos

- Muestra de población adulta

- Artículos publicados entre 2010 - 2020

- Investigaciones pertenecientes al área de estudio de la psicología clínica y farmacología

Por otra parte, los criterios de exclusión fueron los siguientes:

- Tesis o investigaciones de pregrado

- Investigaciones diferentes al idioma inglés y español

- Entrevistas a profesionales

En Scopus se encontraron 15 resultados tras la búsqueda con las palabras claves y mediante los criterios de filtro: artículos entre 2010-2020, artículos de acceso abierto, área de estudio de psicología y farmacología. Por otra parte, Research Gate no muestra el número de registros encontrados en los resultados de búsqueda, por tanto, se procedió a realizar una elección manual en donde se descartaron aquellos artículos que no cumplían con los criterios de inclusión. La búsqueda fue complementada mediante la modalidad bola de nieve, con la finalidad de localizar estudios relevantes no encontrados en primera instancia.

Finalmente, se tomó en consideración un total de 39 artículos, entre los cuales 19 fueron aceptados después de una lectura del título, resumen y muestra en el caso de los estudios clínicos e investigaciones empíricas. De este modo, 8 artículos fueron seleccionados de Scopus, 5 de Research Gate y 6 mediante la modalidad de Bola de nieve.

\section{Resultados}

\subsection{Psicoterapia}

La terapia basada en evidencia acepta modelos tales como: la terapia cognitivo-conductual (TCC), la terapia centrada en la familia y la terapia de ritmos interpersonales y sociales. La TCC se basa en la premisa de que los trastornos del estado de ánimo son consecuencia de 
un pensamiento inadaptado y disfuncional, centrándose en que las cogniciones problemáticas pueden modificarse. La terapia centrada en la familia se basa en la premisa de que la crítica y la hostilidad de los cuidadores contribuyen y aumentan el riesgo de recaída, que puede modificarse cambiando la comunicación entre los miembros de la familia y aumentando las aptitudes para resolver problemas. La terapia de ritmos interpersonales y sociales atribuye los episodios de humor asociados como una perturbación de los ritmos circadianos, que pueden modificarse regulando las rutinas diarias y los ritmos de sueño y vigilia. ${ }^{7}$

\subsubsection{Terapia cognitivo conductual}

La TCC se centra en la modificación de conductas disfuncionales, pensamientos negativos distorsionados, específicamente respecto a la triada cognitiva planteada por Beck, y actitudes desadaptativas relacionadas con la depresión. El terapeuta adopta un rol de educador, buscando la colaboración del paciente, de manera que pueda aprender a reconocer sus patrones de pensamiento negativos y reevaluarlos. Este enfoque requiere que el paciente practique sus nuevas habilidades entre sesiones, mediante tareas y ensayos de nuevas conductas. ${ }^{8}$

En estudios de tipo meta-analítico, entendiendo la TCC como una terapia basada en la evidencia, su eficacia se reporta similar al uso de medicación antidepresiva. ${ }^{9}$ Veleda et al. ${ }^{10}$ demostraron que la TCC es eficaz para la depresión en adultos y que puede aplicarse cuando existe resistencia al tratamiento psicofarmacológico, así mismo el tratamiento complementario mejora significativamente la eficacia del tratamiento. ${ }^{10}$

Puerta y Padilla11 refieren que la TCC, se presenta como la estrategia de tipo no farmacológico por excelencia en el tratamiento de la depresión, definiendo como objetivos en este diagnóstico: disminuir pensamiento disfuncional, incrementar el autocontrol, la capacidad de resolución de problemas y habilidades sociales. ${ }^{11}$ Acevedo y Gélvez ${ }^{12}$ ejemplifican un proceso efectivo de intervención cognitivoconductual de 14 sesiones, utilizando como técnicas la reatribución y conceptualización alternativa, así mismo, fortalecieron el plan de intervención con mediciones pre y post tratamiento. ${ }^{12}$

Mira et al. ${ }^{13}$ proponen el desarrollo de un protocolo basado en la psicología positiva integrada con TCC para el tratamiento de síntomas depresivos.13 Este protocolo se demuestra viable, con mejoría significativa frente a los síntomas depresivos y ansiosos, aumento del afecto positivo, la orientación al disfrute y aceptación del tratamiento.

Rodríguez y González ${ }^{14}$ tras un estudio de caso, refieren que la TCC en conjunto con la terapia centrada en soluciones resulta eficaz en el tratamiento terapéutico del TDM, aplicando técnicas como la relajación muscular, reestructuración cognitiva, entrenamiento en asertividad y habilidades sociales. Los resultados tras la intervención mostraron mejoría importante en los niveles de depresión, así como en el grado de alteración de los pensamientos asociados al trastorno, aumento de asertividad y niveles de autoestima. $^{14}$

Respecto a intervenciones sobre la depresión con diversos tipos de sintomatología asociada, se reporta que las intervenciones psicológicas y farmacológicas tienen una eficacia similar, destacando que el tratamiento combinado podría duplicar el beneficio en comparación con intervenciones individuales. Añadir la TCC al tratamiento psicofarmacológico habitual en pacientes con depresión resistente mejoró significativamente la gravedad de los síntomas, también se recomienda utilizarla como reforzador de adherencia al tratamiento. ${ }^{7}$

En otras presentaciones clínicas, como la psicosis temprana con síntomas depresivos, no se encontró que la TCC fuera más efectiva que los tratamientos convencionales, en la reducción del cuadro depresivo durante la fase activa del primer brote. Sin embargo, la TCC parece mejorar tanto la sintomatología como el funcionamiento en los pacientes durante las fases de síntomas negativos..$^{15}$

En contraste, otra revisión meta-analítica sobre sintomatología psicótica encontró que la TCC demostró ser eficaz para reducir la gravedad de los síntomas, sin embargo, en seis estudios no se encontraron diferencias significativas con otros tratamientos. Su capacidad en contra de la recaída se registró como pobremente eficaz en un conjunto de 21 estudios revisados. ${ }^{16}$

Del mismo modo, Pérez17 realiza una revisión sistemática en la cual afirma que aún cuando las intervenciones psicológicas se utilizan ampliamente y se recomiendan, a menudo tras el fracaso de la medicación en el TDM, falta de evidencia científica sobre su efectividad, lo que pone en relieve la urgente necesidad de realizar estudios con un diseño robusto y controlado sobre esta temática. ${ }^{17}$

\subsubsection{Intervenciones psicosociales}

Las intervenciones de tipo psicosocial, resultan complementarias pues se centran menos en la reducción de los síntomas y más en volver a participar en actividades que mejoren el funcionamiento. Se ha demostrado que reducen las futuras recaídas en depresión y psicosis, manteniendo aceptación y credibilidad desde los pacientes. Estas intervenciones pueden ser utilizadas para apoyar la recuperación a largo plazo en esta población. ${ }^{6}$

Desde un caso clínico de $\mathrm{TDM}^{18}{ }^{18}$ una intervención de activación conductual con técnicas como la relajación, reestructuración cognitiva y entrenamiento en habilidades sociales, reportó mejorías significativas de relaciones familiares e interpersonales, además de un manejo efectivo de emociones y la disminución de los sentimientos de tristeza.18 En consecuencia, se concluye que la aplicación tuvo impacto positivo para el tratamiento de depresión y que las técnicas fueron las adecuadas para poder responder a las demandas presentadas.

Romero, Poves, y Vucinovich, ${ }^{19}$ reportaron que la activación conductual resulta efectiva para el tratamiento de un trastorno psicótico no especificado con sintomatología de- 
presiva, el estudio se centró en: regulación de higiene del sueño e incremento en la calidad de relaciones sociales, además se destaca la importancia del aprendizaje de habilidades de autocuidado.19 Se concluye que la intervención ha sido eficaz para disminuir la sintomatología depresiva y psicótica, considerando que los mismos procesos psicológicos se expresan como factores de mantenimiento.

\subsubsection{Otras intervenciones psicológicas}

Se debe considerar que se han encontrado varios mecanismos neurobiológicos que pueden contribuir a la disfunción cognitiva en TDM, incluyendo la sobre-activación del eje hipotalámico-pituitario-suprarrenal, desequilibrios en las vías de la supervivencia y muerte celular. La disfunción cognitiva en el TDM abarca varios dominios, como las funciones ejecutivas, memoria verbal y atención. Además de ser una manifestación frecuente, puede persistir durante la fase de remisión, por esto, se debe disponer de un protocolo de contingencia en rehabilitación cognitiva para cuando estos síntomas se manifiesten. ${ }^{20}$

Carrasco21 por su parte, plantea la posibilidad de integrar técnicas y principios de diversos enfoques psicológicos, psicodinámicos y humanistas, con el objetivo de poder utilizar cualquier técnica de otro modelo, partiendo de la condición de que existan puntos comunes sobre la patología y su tratamiento. 21

\subsection{Farmacoterapia}

La fisiopatología de la depresión es compleja y, en parte, todavía enigmática. En su desarrollo contribuyen múltiples factores biológicos, genéticos, de estructura y función cerebral, mecanismos inflamatorios y endocrinológicos. ${ }^{1}$ Por esto es importante que cualquier abordaje incluya los principios establecidos por las ciencias biológicas, específicamente los tratamientos farmacológicos.
González et al., ${ }^{2}$ realizan una actualización de los trastornos depresivos sobre factores etiopatogénicos, manifestaciones clínicas, diagnóstico diferencial, pronóstico, complicaciones y comorbilidad. ${ }^{2}$ Respecto al subtipo de TDM con síntomas psicóticos establecen el uso de antidepresivos y antipsicóticos entre 12 a 24 meses; en caso de resistencia, alto riesgo o agitación severa, el tratamiento de primera línea es la terapia electro convulsiva. El uso de antidepresivos sumado con antipsicóticos de segunda generación, se ha evidenciado como un tratamiento efectivo, con adecuada adherencia al tratamiento y mantenimiento de sus efectos, significativamente superior a la monoterapia con antidepresivos. $^{22}$

El desarrollo de nuevos tratamientos farmacológicos, incluye opioides, psicodélicos, ketamina e inclusive cannabinoides, además de los constantes intentos por perfeccionar las opciones ya disponibles. Sin embargo, ante las condiciones propias de cada contexto, se vuelve perentorio optimizar el uso de las opciones farmacológicas que están disponibles en el contexto del paciente. Existen varios medicamentos psicotrópicos disponibles, pero lograr una respuesta terapéutica óptima resulta un verdadero desafío. El TDM y los síntomas psicóticos representan fenómenos heterogéneos y las respuestas a los medicamentos varían entre pacientes. ${ }^{3}$

\subsection{Planificación de la intervención psicológica}

Con base en lo expuesto, resulta necesario estructurar una guía para las intervenciones psicoterapéuticas recomendadas en TDM con síntomas psicóticos. Las técnicas se aplican en función de las problemáticas relevantes expresadas por cada caso particular. Y pueden estructurarse en función de las necesidades y avance del caso, las técnicas se detallan en la Tabla 1.

Tabla 1: Planificación psicoterapéutica del trastorno depresivo mayor con síntomas psicóticos

\begin{tabular}{|c|c|c|c|c|}
\hline Técnicas & Ef* & Problemas relevantes & Objetivo de la técnica & Descripción \\
\hline Psicoeducación & CG & $\begin{array}{l}\text { Adherencia al tratamiento } \\
\text { Ausencia de conciencia de en- } \\
\text { fermedad y necesidad de trata- } \\
\text { miento }\end{array}$ & $\begin{array}{l}\text { Explicar la fundamentación de la TCC } \\
\text { Generar adherencia al tratamiento farma- } \\
\text { cológico y psicológico } \\
\text { Fomentar la motivación al cambio }\end{array}$ & $\begin{array}{l}\text { Mediante un abordaje directivo explicar } \\
\text { la naturaleza de la enfermedad, formas } \\
\text { de intervención y condiciones del trata- } \\
\text { miento }\end{array}$ \\
\hline $\begin{array}{l}\text { Reestructuración } \\
\text { cognitiva }\end{array}$ & CG & $\begin{array}{l}\text { Estado de ánimo deprimido } \\
\text { Sentimientos de } \\
\text { inutilidad/culpa inapropiada } \\
\text { Ideación suicida } \\
\text { Triada cognitiva alterada }\end{array}$ & $\begin{array}{l}\text { Adquirir formas más adaptativas o fle- } \\
\text { xibles en su estructura cognitiva elimi- } \\
\text { nando aquellos pensamientos automáti- } \\
\text { cos desadaptativos que están en la base } \\
\text { del espectro depresivo }\end{array}$ & $\begin{array}{l}\text { Aprendizaje y clasificación de las distor- } \\
\text { siones cognitivas } \\
\text { Registro del pensamiento } \\
\text { Cuestionamiento/evaluación de las dis- } \\
\text { torsiones } \\
\text { Flecha descendente } \\
\text { Cuestionamiento socrático } \\
\text { Análisis y evaluación empírica } \\
\text { Aprendizaje de formas alternativas de } \\
\text { pensamiento }\end{array}$ \\
\hline $\begin{array}{l}\text { Entrenamiento } \\
\text { en resolución } \\
\text { de problemas }\end{array}$ & $\begin{array}{l}\text { CG } \\
\text { CC }\end{array}$ & $\begin{array}{l}\text { Manejo inefectivo de estreso- } \\
\text { res recientes y situaciones li- } \\
\text { gadas al mantenimiento }\end{array}$ & $\begin{array}{l}\text { Estructurar nuevas formas de actuación } \\
\text { efectivas y adaptativas frente a diversas } \\
\text { situaciones vitales presentes y que pue- } \\
\text { dan surgir a futuro }\end{array}$ & $\begin{array}{l}\text { Recolección de información } \\
\text { Definición del problema } \\
\text { Generación de alternativas } \\
\text { Toma de decisiones } \\
\text { Verificación de resultados }\end{array}$ \\
\hline
\end{tabular}


Tabla 1: Planificación psicoterapéutica del trastorno depresivo mayor con síntomas psicóticos...(continuación)

\begin{tabular}{|c|c|c|c|c|}
\hline Técnicas & Ef* & Problemas relevantes & Objetivo de la técnica & Descripción \\
\hline Psicoeducación & $\begin{array}{l}\text { CG } \\
\mathrm{CC}\end{array}$ & $\begin{array}{l}\text { Alteración del sueño } \\
\text { Agitación psicomotora }\end{array}$ & $\begin{array}{l}\text { Reducir la activación fisiológica elevada } \\
\text { por medio de técnicas de respiración y } \\
\text { relajación. } \\
\text { Centrar al paciente en la realidad pa- } \\
\text { ra desligarlo de su condición para que } \\
\text { consiga conciliar de forma adecuada el } \\
\text { sueño. }\end{array}$ & $\begin{array}{l}\text { Respiración al cuadrado } \\
\text { Variante de la relajación progresiva de } \\
\text { Jacobson }\end{array}$ \\
\hline $\begin{array}{l}\text { Entrenamiento } \\
\text { en habilidades } \\
\text { sociales: } \\
\text { asertividad, } \\
\text { manejo social, } \\
\text { empatía }\end{array}$ & $\begin{array}{l}\text { CG } \\
\text { CC }\end{array}$ & $\begin{array}{l}\text { Funcionamiento social pertur- } \\
\text { bado }\end{array}$ & $\begin{array}{l}\text { Mejorar destrezas sociales deficientes o } \\
\text { conflictivas favoreciendo la asertividad, } \\
\text { la convivencia y aumentar la cantidad de } \\
\text { apoyo }\end{array}$ & $\begin{array}{l}\text { Role-playing para ensayar situaciones } \\
\text { sociales difíciles para el paciente para ser } \\
\text { extrapoladas a situaciones reales }\end{array}$ \\
\hline $\begin{array}{l}\text { Entrenamiento } \\
\text { en técnicas de } \\
\text { autocontrol }\end{array}$ & $\begin{array}{l}\text { CG } \\
\mathrm{CC}\end{array}$ & $\begin{array}{l}\text { Pensamientos catastróficos e } \\
\text { ideación suicida }\end{array}$ & $\begin{array}{l}\text { Reducir la tendencia a responder impul- } \\
\text { sivamente para pensar la situación en- } \\
\text { contrando una solución razonable }\end{array}$ & $\begin{array}{l}\text { Técnica de reatribución } \\
\text { Conceptualización alternativa }\end{array}$ \\
\hline $\begin{array}{l}\text { Activación } \\
\text { conductual/ } \\
\text { Programa de } \\
\text { actividades } \\
\text { agradables }\end{array}$ & $\mathrm{CC}$ & $\begin{array}{l}\text { Anhedonia } \\
\text { Actividad reducida } \\
\text { Incumplimiento de sus activi- } \\
\text { dades diarias }\end{array}$ & $\begin{array}{l}\text { Establecer actividades o estrategias sim- } \\
\text { ples que le mantengan activo durante el } \\
\text { día } \\
\text { Incrementar la frecuencia de actividades } \\
\text { sociales placenteras } \\
\text { Reinsertar al paciente en sus actividades } \\
\text { cotidianas y laborales }\end{array}$ & $\begin{array}{l}\text { Consiste en monitorear las actividades } \\
\text { evaluándolas en función del placer y do- } \\
\text { minio para asignar actividades inter se- } \\
\text { sión. Además, reinsertarle en sus tareas } \\
\text { diarias en las diversas áreas de funciona- } \\
\text { miento del paciente }\end{array}$ \\
\hline
\end{tabular}

Fuente: Elaboración propia

Nota: ${ }^{*} \mathrm{EF}=$ Enfoque; $\mathrm{CG}=\mathrm{Cognitiva} ; \mathrm{CC}=$ Conductual

\section{Conclusiones y consideraciones al tratamiento}

El plan terapéutico presentado previamente representa un conjunto de técnicas diversas fundamentadas en la TCC, que se proponen ser aplicadas de acuerdo a las necesidades específicas que cada caso amerite. Es fundamental que cualquier intervención psicológica se desarrolle en sintonía con la evolución del tratamiento farmacológico dentro de un marco de colaboración con el equipo multidisciplinario.

El tratamiento farmacológico, a rasgos generales contempla la administración conjunta, en periodos de entre 12 a 24 meses en una fase inicial de antidepresivos y antipsicóticos. La dosificación de estos últimos disminuye conforme ceden las alucinaciones y delirios. La prescripción farmacológica ideal implicaría el uso de antidepresivos inhibidores selectivos de la recaptación de serotonina (ISRS) como la Fluoxetina y antipsicóticos de segunda generación como la Quetiapina, pues tienen aplicaciones y principios antidepresivos. Sin embargo, la farmacopea local y las condiciones de uso están sujetos a la disponibilidad.

De acuerdo con la literatura, cuando desaparece la sintomatología psicótica es el momento adecuado para iniciar un tratamiento psicoterapéutico con objetivos concretos fundamentados en las capacidades que presenta el paciente. Durante la fase previa, la intervención psicológica está centrada en el acompañamiento del paciente y su familia, del mismo modo, se enfoca en evaluar la evolución de los síntomas en un proceso de comunicación activa con el psiquiatra.

En conclusión, un abordaje eficaz del TDM con síntomas psicóticos implica un tratamiento combinado. La TCC es la principal forma de intervención fundamentada en la evidencia que se demuestra eficaz por la literatura académica disponible. El tratamiento farmacológico se fundamenta en el uso de antidepresivos ISRS y antipsicóticos de segunda generación, regulando los niveles de serotonina, noradrenalina y dopamina.

\section{Fuente de Financiamiento}

Este estudio es autofinanciado.

\section{Conflicto de Intereses}

No existen conflictos personales, profesionales, financieroso de otro tipo.

\section{Consentimiento Informado}

Los autores cuentan con el consentimiento informado de los pacientes para la investigación, la publicación del caso y sus imágenes. 


\section{Referencias Bibliográficas}

1. Álvarez, M., Pereira, V., \& Ortuño, F. Tratamiento de la depresión. Medicine. 2017:12(4), 2731-2742.

2. González, X., Martín, M., Sánchez, M. \& Mirapeix, I. Trastornos del humor: trastornos depresivos. Medicine. 2015:11(85), 64-74.

3. Ceskova, E., \& Silhan, P. Novel treatment options in depression and psychosis. Neuropsychiatric Disease and Treatment. 2018; 741-747.

4. American Psychological Association. Manual diagnóstico y estadístico de los trastornos mentales. DSM-5. Bogotá: Medica Panamericana; 2014.

5. Ministerio de Salud Pública del Ecuador. Diagnóstico y 17. tratamiento del episodio depresivo y del trastorno depresivo recurrente en adultos. Guía Práctica Clínica. Ecuador: Ministerio de Salud Pública del Ecuador; 2017.

6. Gaudiano, B., Busch, M., Wenze, S., Nowlan K., Epstein, G., \& Miller, I. Acceptance-based Behavior Therapy for Depression With Psychosis: Results From a Pilot Feasibility Randomized Controlled Trial. Journal of Psychiatric Practice. 2015; (21), 320-332.

7. O'Brien, O., Lee, D., Swann, A., Mathew, S., \& Lijffijt, M. Psychotherapy for Mixed Depression and Mixed Mania. Psichiatric Clinics of North America. 2020; (43), 199-211.

8. Grupo de trabajo de la Guía de Práctica Clínica sobre el Manejo de la Depresión en el Adulto. Guía de Práctica Clínica sobre el Manejo de la Depresión en el Adulto. Galicia: Ministerio de Sanidad, Servicios Sociales e Igual- 2 dad. Agencia de Evaluación de Tecnologías Sanitarias de Galicia; 2014.

9. Sanz, J., \& García, M. Ideas equivocadas sobre la depre- 22 sión y su tratamiento. Papeles del Psicólogo. 2017:38(3).

10. Veleda, G., Lopes, M., Silva, R., Jansen, K., Neufeld, C., \& Souza, L. Effect of cognitive- behavioral therapy after treatment for adults with major depression. Psicología: Teoría e Práctica. 2019:21(3), 345-365

11. Puerta, J., \& Padilla, D. Terapia cognitiva-conductual (TCC) Como tratamiento para la depresión: Una revisión del estado del arte. Revista de la Faculta de Ciencias de la Salud de la Universidad de Magdalena. 2011; (2), 251257.

12. Acevedo, M., \& Gélvez, L. Estrategias de intervención cognitivo conductual en un caso de depresión persistente. Revista Virtual Universidad Católica del Norte. 2018:55(1), 156-158.

13. Mira, A., Enrique, A., Díaz, A., Rachyla, I., González, A., Brentón, J., \& Botella, C. Viabilidad de una intervención psicológica clínica positiva para síntomas depresivos: Una serie de casos. Revista Argentina de Clínica Psicológica. 2018:27(3), 381-392.

14. Rodríguez, D., \& González, M. Integración de la terapia cognitivo conductual y la terapia centrada en soluciones en un caso de depresión mayor. Revista Electrónica de Psicología Iztacala. 2020:22(1), 147-179.

15. Sönmez, N., Romm, K., Østefjells, T., Grande, M., HunnickE L., Hummelen, B., Tesli, M., Melle, I., \& Røssberg, J. Cognitive behavior therapy in early psychosis with a focus on depression and low self-esteem: A randomized controlled trial. Comprehensive Psychiatry. 2019.

16. Jauhar, S., McKenna, P., Radua, J., Fung, E., Salvador, R., \& Laws, K. Cognitive-behavioural therapy for the symptoms of schizophrenia: systematic review and metaanalysis with examination of potential bias. The British Journal of Psychiatry. 2014; (204), 20-29.

17. Pérez, A. La depresión resistente al tratamiento farmacológico y/o psicológico en adultos: una revisión sistemática. Universidad de la Laguna; 2013.

18. Salinas, J. El tratamiento de la depresión con un enfoque de activación conductual: Un caso. Revista Electrónica de Psicología Iztacala. 2010:13(3), 105-126.

19. Romero, R., Poves, S., \& Vucinovich, N. Efectos de la activación conductual sobre la sintomatología depresiva, la sintomatología psicótica y el nivel de discapacidad percibida: a propósito de un caso. Análisis y Modificación de Conducta. 2015:143(45), 59-73

0. Bortolato, B., Miskowiak, K., Köhler, C., Maes, M.., Fernandes, B., Berk, M., \& Carvalho, A. Cognitive remission: a novel objective for the treatment of major depression? BMC Medicine, 2016:14(9).

21. Carrasco, A. Modelos psicoterapéuticos para la depresión: hacia un enfoque integrado. Interamerican Journal of Psychology. 2017:51(2), 181-189.

22. Kim, D., Rascati, K., Richards, K., Ford, K., Wilson, J., \& Beretvas, S. Medication Adherence and Persistence in Patients with Severe Major Depressive Disorder with Psychotic Features: Antidepressant and Second-Generation Antipsychotic Therapy Versus Antidepressant Monotherapy. Journal of managed care \& specialty pharmacy. 2016:22.

Recibido: 10 de mayo de 2020

Aceptado: 5 de octubre de 2020 